\title{
Observações comportamentais de ovinos em pastejo no bioma Cerrado do Distrito Federal
}

\section{Behavioral observations of grazing sheep in the Cerrado biome of Federal District}

\author{
David Germano Gonçalves Schwarz ${ }^{1 *}$; Carlos Thiago Silveira Alvim Mendes \\ de Oliveira²; Flávia Aline Silveira Alvim Mendes de Oliveira ${ }^{1}$; Juaci Vitória Malaquias ${ }^{3}$
}

\section{Resumo}

O conhecimento do comportamento animal sob pastejo em diferentes biomas é uma alternativa para o incremento na produção, pois identifica possibilidades de intervenções estratégicas no manejo. O objetivo do presente trabalho foi avaliar se o tempo médio de pastejo, ruminação e ócio estão relacionados com a posição (estação/decúbito), com o local (sob sol ou sombra), bem como a influência do sexo nestas interações em ovinos da raça Santa Inês no bioma Cerrado do Distrito Federal. Para a obtenção dos dados, o comportamento dos animais foi avaliado por observações visuais em intervalos de dez minutos num período de dez horas, em dois dias consecutivos. A partir dos dados obtidos foram realizadas as análises de variância e comparações entre as médias pelo teste de Tukey com nível de significância de $5 \%$. O resultado deste estudo permitiu concluir que os tempos médios de pastejo, ruminação e ócio nos ovinos da raça Santa Inês, estão relacionados com a posição e o local. Não foram identificadas diferenças significativas em relação ao tempo gasto na atividade de pastejo, ruminação e ócio entre machos e fêmeas.

Palavras-chave: Cerrado, etologia, gênero, pequenos ruminantes, Santa Inês

\begin{abstract}
The knowledge of animal behavior under grazing in different biomes is an alternative to increase production because it identifies possibilities of strategic interventions in the management. The aim of this study was to evaluate if the average time of grazing, ruminating and leisure are related to the position (station/decubitus), to the location (sun or shade), as well as the influence of gender in these interactions in Santa Inês sheep in Cerrado from Federal District, Brazil. To obtain the data, the behavior of the animals was assessed by visual observations at ten-minute intervals over a period of ten hours on two consecutive days. From the data obtained were analysis of variance and comparisons between means by Tukey test with significance level of 5\%. The result of this study showed that the average time of grazing, ruminating and leisure in Santa Inês sheep, are related to the position (station/decubitus) and location (sun and shade). No significant differences were identified in relation to time spent in the activity of grazing, ruminating and leisure between Santa Inês sheep males and females.
\end{abstract}

Key words: Cerrado, ethology, gender, small ruminants, Santa Inês

\footnotetext{
${ }^{1}$ Pós-graduando em Medicina Veterinária, Universidade Federal de Viçosa, UFV, Viçosa, MG. E-mail: davidggs@yahoo.com.br; flavialvimvet@hotmail

${ }^{2}$ Pós-graduando em Zootecnia, UFV, Viçosa, MG. E-mail: ctsamo@gmail.com

${ }^{3}$ Analista em Estatística, Embrapa Cerrados, Planaltina, DF. E-mail: juaci.malaquias@cpac.embrapa.br

* Autor para correspondência
} 
O Cerrado ocupa cerca de $23,92 \%$ de toda área do Brasil, sendo encontrado nos estados de Tocantins, Maranhão, Minas Gerais, Goiás, Mato Grosso do Sul e todo o Distrito Federal. Devido sua grande abrangência territorial é considerado o segundo maior bioma do país, sendo menor apenas ao bioma Amazônia (IBGE, 2004). Nessa região, $26,5 \%$ da atividade que institui o uso da terra são destinados à pastagens cultivadas, caracterizadas por distribuições heterogêneas em toda sua extensão (SANO et al., 2008).

O conhecimento do uso dessas regiões na produção animal é fator importante para o estabelecimento de possíveis alterações no comportamento em diferentes espécies animais. Além disso, o desempenho de animais em pastejo é influenciado por vários fatores associados ao animal, ao pasto, ao ambiente e às suas interações (CARVALHO et al., 2007). Nesse sentido, a ampla distribuição mundial da espécie ovina, relacionase à sua capacidade de adaptação em diferentes condições climáticas e alimentares (CUNHA et al., 1997).

Por meio do entendimento comportamental dos animais em pastejo podem-se determinar intervenções estratégicas no manejo, bem como aperfeiçoar ações corretivas para o melhor aproveitamento das pastagens nas condições ambientais em que os animais estão inseridos. Portanto, o conhecimento do tempo de pastejo por meio do estudo dos ciclos diários é de fundamental importância nesses sistemas de produção à pasto (RIBEIRO FILHO et al., 1997). De acordo com Penning (2004), quando não se faz uso de registros automáticos das atividades dos animais, a observação visual dos mesmos em intervalos de 5 a 10 minutos permite atingir níveis adequados de acurácia. Dessa forma, além das atividades de pastejo, outras atividades como ruminação e ócio podem ser analisadas.

Tendo verificado a importância do estudo comportamental de animais em pastejo inseridos em diferentes biomas, foi realizado um trabalho preliminar com o objetivo de avaliar a influência do tempo médio de pastejo, ruminação e ócio em relação à posição (em estação/decúbito), sob sol ou sombra, bem como a influência do sexo nestas interações em ovinos da raça Santa Inês no bioma Cerrado do Distrito Federal.

O estudo foi conduzido na Granja Nova Cambuci, localizada em Sobradinho, cidade Satélite de Brasília-DF, a $20 \mathrm{Km}$ do Plano Piloto. O experimento foi realizado em Junho de 2009 utilizando nove ovinos adultos da raça Santa Inês (3 machos e 6 fêmeas) por tratamento, ou seja, nove repetições. Foram realizadas duas observações em dias consecutivos, com duração de $10 \mathrm{~h} /$ dia (07h às 17h).

Os animais foram presos as $18 \mathrm{~h}$ e mantidos em abrigos durante a noite, sendo soltos as $07 \mathrm{~h}$ do dia seguinte em piquete de aproximadamente $4000 \mathrm{~m}^{2}$, constituídos por Brachiaria ruziziensis, providos de sal mineral, água e sombra natural. O ensaio compreendeu período de adaptação de 15 dias antes do início das observações. Durante o experimento os animais não receberam alimentação à base de concentrado.

O experimento foi realizado com delineamento inteiramente casualizado em esquema fatorial $3 \times 2 \times 2 \times 2$. No qual, as variáveis analisadas foram: situação (tempo de pastejo, tempo de ruminação e tempo de ócio), local (tempo sob sol ou tempo sob sombra), posição (tempo em estação ou tempo em decúbito) e sexo (macho e fêmea),

Os hábitos em pastejo foram obtidos por meio de observações visuais dos animais a cada 10 minutos, sendo o tempo total o somatório das vezes nas quais os animais foram observados em determinado estado. Para esse estudo considerou-se que a atitude anotada a cada momento de observação foi mantida durante os dez minutos seguintes.

Com os dados coletados foram realizadas as análises de variância, sendo as diferenças entre as médias sequencialmente avaliadas pelo teste de 
Tukey para nível de significância de 5\%. Todas as análises foram processadas pelo programa estatístico SAS versão 9.1 (SAS INSTITUTE, 2002).

$\mathrm{Na}$ Figura 1 podem ser observados o tempo médio em minutos e desvio padrão (DP) do comportamento dos animais em pastejo $(113,47$ min./DP:165,84), ócio (20,83 $\mathrm{min} . / \mathrm{DP}: 19,88)$ e ruminação (18,19 min./DP:26,44). Observouse que o tempo de pastejo foi superior ao de ócio e ruminação. No entanto, não houve diferença significativa entre o tempo de ruminação e de ócio. Lin et al. (2011) verificaram comportamento semelhante em ovinos, onde aproximadamente 50\% de todo tempo diurno foi destinado exclusivamente ao pastejo. Esse aumento do tempo de pastejo pode ser, em parte, explicado pelo manejo aplicado aos animais, no qual o fato de permanecerem confinados durante a noite implicaria no aumento do tempo de apreensão e busca do alimento durante o dia. Essas informações corroboram com os resultados de Cunha et al. (1997) que identificaram comportamento semelhante em ovelhas manejadas com restrição no pastejo, pois, de acordo com os mesmos autores, o fato de limitar o tempo de pastejo dos ovinos, os força a permanecerem mais tempo buscando alimento durante o dia, mesmo em horários mais críticos de temperatura.

Figura 1. Tempo médio em minutos e desvio padrão (DP) para cada situação (pastejo, ruminação e ócio) em ovinos da raça Santa Inês no bioma Cerrado.

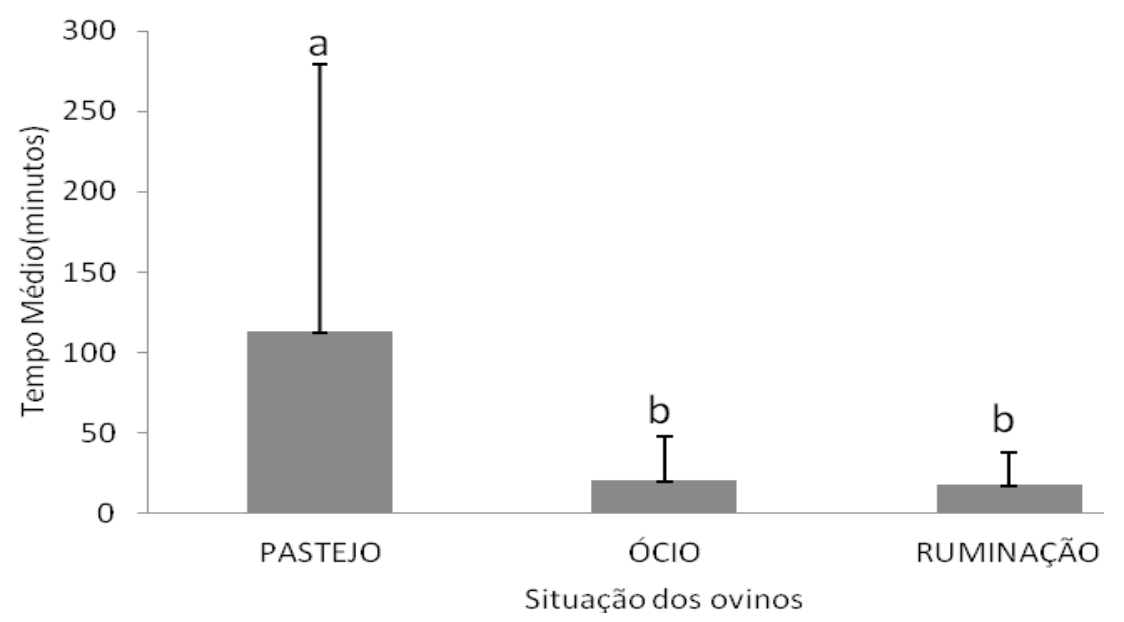

Fonte: Elaboração dos autores.

Em relação ao tempo em ócio, Leoni et al. (2006) verificaram que o aumento do tempo em ócio de ovinos alimentados com feno, somente foi verificada com a inclusão de $40 \%$ de concentrado na dieta. Como nesse estudo os animais não receberam suplementação, pode-se inferir que a dieta exclusivamente constituída de volumoso aumentou o tempo de ingestão e reduziu o tempo gasto em outras atividades.

$\mathrm{Na}$ Figura 2 pode ser observado o tempo médio em minutos do comportamento das fêmeas em pastejo (114,37min/DP: 166,39), ruminação $(17,92 \mathrm{~min} / \mathrm{DP}: 20,73)$ e ócio $(20,21 \mathrm{~min} / \mathrm{DP}: 26,46)$ e dos machos em pastejo (111,67min/DP:168,28), ruminação $(18,75 \mathrm{~min} / \mathrm{DP}: 18,49)$ e ócio $(22,08 \mathrm{~min} /$ DP:27,82). Na situação pastejo, o tempo médio das fêmeas (114,37min.) foi maior comparado ao dos machos (111,67min.), no entanto, nenhuma das interações: situação (pastejo, ruminação e ócio) vs sexo teve significância estatística. Esses resultados são distintos dos encontrados por Parente et al. (2007) que observaram o aumento no tempo 
de pastejo para machos (borregos) em relação às fêmeas (borregas e ovelhas). Contudo, sabe-se que as fêmeas podem modificar seu comportamento ingestivo dependendo do estado fisiológico em que se encontram (gestação, amamentação, etc).

No presente estudo, independentemente do gênero, os animais permaneceram em média 71,94 minutos sob sol e 29,72 minutos à sombra. Provavelmente esse comportamento ocorreu devido à maior rusticidade da raça Santa Inês à incidência solar. Pois, segundo experimento realizado por Cezar et al. (2004), tanto os machos como as fêmeas dessa raça mantiveram valores de taxas cardíacas e respiratórias inferiores às demais raças estudadas, quando em manejo semi-extensivo. Embora o presente estudo tenha demonstrado maior tempo de permanência dos animais sob o sol, em determinados períodos do dia houve a busca por sombra. Nesse sentido, Cunha et al. (1997) reforçaram em seu estudo a necessidade de implementação no manejo e no fornecimento de áreas sombreadas aos animais, uma vez que a temperatura e a umidade estão associadas ao comportamento dos animais em pastejo e na procura de sombra.

Figura 2. Tempo médio em minutos do comportamento de ovinos da raça Santa Inês machos e fêmeas sob pastejo, ruminação e ócio, no bioma Cerrado do Distrito Federal.

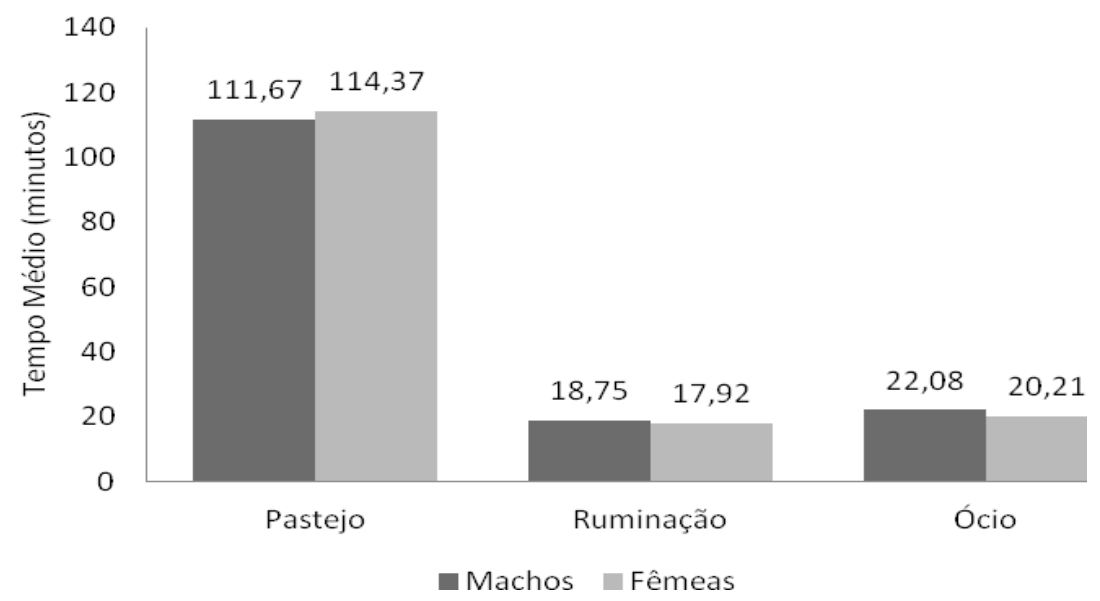

Fonte: Elaboração dos autores.

O tempo elevado de pastejo dos ovinos influenciou significativamente na posição desses animais, demonstrando a permanência de maior tempo em estação (96,94 minutos), em relação ao decúbito (4,72 minutos), pela busca de forragem.

Considerando as possíveis interações entre as variáveis analisadas foram constatadas significância estatística para três interações de segunda ordem: situação $v s$ local, situação $v s$ posição e local $v s$ posição.

$\mathrm{Na}$ situação vs local, observou-se que houve interação significativa $(\mathrm{P}<0,05)$ entre pastejo ao sol e pastejo à sombra, demonstrando que o tempo médio de permanência dos ovinos sob o sol foi maior durante o pastejo. Apesar do tempo de ruminação não ter apresentado diferenças significativas entre as médias dos tempos em que os animais permaneceram em cada uma dessas posições (sol ou sombra), observou-se que o tempo de ruminação à sombra (29,17 minutos) foi superior ao de ruminação ao sol (7,22 minutos).

Considerando a interação das médias entre as variáveis situação vs posição, houve diferença significativa entre o tempo médio em que os animais permaneceram em decúbito e em estação para as 
categorias de situações (pastejo e ócio), sendo os valores superiores para o ócio (40,3 minutos) e o pastejo (226,9 minutos) em estação. O maior tempo de pastejo em relação ao ócio pode estar relacionado à capacidade seletiva dos ovinos. Nesse sentido, a busca por partes mais tenras da forragem elevaria o tempo de pastejo e consequentemente o tempo médio em que os animais permaneceriam em estação nessa atividade. Por outro lado, Lin et al. (2011) relatam que os ovinos podem aumentar o tempo de pastejo em detrimento do tempo de repouso para compensar situações de reduzida disponibilidade de forragem.

Para a relação do local vs posição foi constatado que o tempo médio pelo qual o animal permaneceu em estação foi superior ao tempo médio em que estes permaneceram em decúbito, independentemente do local (sombra ou sol). Marques et al. (2006) constataram resultados semelhantes em bovinos, no qual os animais permaneceram mais tempo em estação do que em decúbito quando no período de descanso, relacionando a posição dos animais ao índice pluviométrico da região estudada.

Em relação à análise tríplice da variância foi demonstrado correlações estatisticamente significativas entre as variáveis: situação vs local vs posição. Foram observados que os animais ao estarem em ócio ficaram mais tempo em estação e à sombra. Outrossim, quando da ruminação, estes também permaneceram à sombra e em estação. Em relação aos resultados obtidos por Marques et al. (2006) na espécie bovina, verificaram maior permanência de animais em decúbito ao ruminar. Por fim, quando se referiu à posição em pastejo, estes ficaram em estação e preferencialmente sob o sol por maior tempo.

Verificou-se que os ovinos estudados apresentaram comportamento preferencial por: pastejo sob sol e em estação; ruminação à sombra e em estação; e ócio à sombra e em estação. Além disso, não foram identificadas diferenças significativas em relação ao tempo gasto na atividade de pastejo, ruminação e ócio entre ovinos Santa Inês machos e fêmeas. Acredita-se que este trabalho seja o primeiro relato do comportamento ovino da raça Santa Inês nestas condições estudadas, onde foi possível relacionar o tempo médio de pastejo, ruminação e ócio com o local e a posição dos ovinos inseridos no bioma Cerrado do Distrito Federal.

\section{Agradecimentos}

Os autores agradecem à Granja Nova Cambuci por ceder espaço e os animais para esse trabalho preliminar do comportamento ovino no Cerrado do Distrito Federal.

\section{Referências}

CARVALHO, P. C. F.; KOZLOSKI, G. V.; RIBEIRO FILHO, H. M. N.; REFFATTI, M. V.; GENRO, T. C. M.; EUCLIDES, V. P. B. Avanços metodológicos na determinação do consumo de ruminantes em pastejo. Revista Brasileira de Zootecnia, Viçosa, MG, v. 36, p. 151-170, 2007. Suplemento Especial.

CEZAR, M. F.; SOUZA, B. B.; SOUZA, W. H.; PIMENTA FILHO, E. C.; TAVARES, G. P.; MEDEIROS, G. X. Avaliação de parâmetros fisiológicos de ovinos Dorper, Santa inês e seus mestiços perante condições climáticas do trópico semi-árido nordestino. Ciência e Agrotecnologia, Lavras, v. 28, n. 3, p. 614-620, 2004.

CUNHA, E. A.; SANTOS, L. E.; RODA, D. S.; POZZI, C. R.; OTSUK, I. P.; BUENO, M. S.; RODRIGUES, C. F. C. Efeito do sistema de manejo sobre o comportamento em pastejo, desempenho ponderal e infestação parasitária em ovinos suffolk. Pesquisa Veterinária Brasileira, Seropédica, v. 17, n. 3-4, p. 105-111, 1997.

INSTITUTO BRASILEIRO DE GEOGRAFIA E ESTATÍSTICA - IBGE. Mapa de biomas do Brasil. Escala 1:5.000.000. Rio de Janeiro: IBGE, 2004. Disponível em: <http://mapas.ibge.gov.br/biomas2/ viewer.htm.>. Acesso em: 13 fev. 2007.

LEONI, E. F.; FREGONESI, J. A.; MIZUBUTI, I. Y.; SALMAZO, R.; ALVES, T. C.; CASIMIRO, T. R. Consumo e digestibilidade aparente de rações com diferentes proporções de feno de capim Coastcross (Cynodon dactylon (L.) Pers.) e soja crua moída e comportamento alimentar de ovinos. Semina: Ciências Agrárias, Londrina, v. 27, n. 4, p. 685-694, 2006. 
LIN, L.; DICKHOEFER, U.; MÜLLER, K.; WURINA, SUSENBETH, A. Grazing behavior of sheep at different stocking rates in the Inner Mongolian steppe, China. Applied Animal Behaviour Science, Amsterdam, v. 129, n. 1, p. 36-42, 2011.

MARQUES, J. A.; NETO, S. F. C.; GROFF, A. M.; SIMONELLI, S. M.; CORASA, J.; ROMERO, L.; ZAWADSKI, F.; ARAÚJO, P. F. Comportamento de bovinos mestiços em confinamento com e sem acesso a sombra durante o período de verão. Campo Digital, Campo Mourão, v. 1, n. 1, p. 54-59, 2006.

PARENTE, H. N.; ZANINE, A. M.; SANTOS, E. M.; FERREIRA, D. J.; OLIVEIRA, J. S. Comportamento ingestivo de ovinos em pastagem de tifton-85 (Cynodon spp) na região Nordeste do Brasil. Revista Ciência Agronômica, Fortaleza, v. 38, n. 2, p. 210-215, 2007.
PENNING, P. D. Animal-based techniques for estimating herbage intake. In: (Ed.). Herbage intake handbook. 2. ed. Reading: The British Grassland Society, 2004. p. 53-94.

RIBEIRO FILHO, H. M. N.; ALMEIDA, E. X.; HARTHMANN, O. E. L.; MARASCHIN, G. E. Tempo e ciclos diários de pastejo de bovinos submetidos a diferentes ofertas de capim-elefante anão cv.'Mott'. In: REUNIÃO ANUAL DA SOCIEDADE BRASILEIRA DE ZOOTECNIA, 34., 1997, Juiz de Fora. Anais... Minas Gerais: Sociedade Brasileira de Zootecnia, 1997. p. 276-278.

SANO, E. E.; ROSA, R.; BRITO, J. L. S.; FERREIRA, L. G. Mapeamento semidetalhado do uso da terra do Bioma Cerrado. Pesquisa Agropecuária Brasileira, Brasília, v. 43, n. 1, p. 153-156, 2008.

SAS Institute. SAS/STAT ${ }^{\circledR}$. User's guide: statistics, version 9.1. Cary: SAS Institute, 2002 